\title{
Development of Next Generation Lifetime PSP Imaging Systems
}

\author{
A. Neal Watkins, Jeffrey D. Jordan \& Bradley D. Leighty \\ NASA Langley Research Center \\ Hampton, VA 23681
}

\author{
JoAnne L. Ingram \& Donald M. Oglesby \\ Swales Aerospace \\ Hampton, VA 23681
}

\begin{abstract}
$\underline{\text { ABSTRACT }}$
This paper describes a lifetime PSP system that has recently been developed using pulsed light-emitting diode (LED) lamps and a new interline transfer CCD camera technology. This system alleviates noise sources associated with lifetime PSP systems that use either flash-lamp or laser excitation sources and intensified CCD cameras for detection. Calibration curves have been acquired for a variety of PSP formulations using this system, and a validation test was recently completed in the Subsonic Aerodynamic Research Laboratory (SARL) at Wright-Patterson Air Force Base (WPAFB). In this test, global surface pressure distributions were recovered using both a standard intensity-based method and the new lifetime system. Results from the lifetime system agree both qualitatively and quantitatively with those measured using the intensity-based method. Finally, an advanced lifetime imaging technique capable of measuring temperature and pressure simultaneously is introduced and initial results are presented.
\end{abstract}

\section{INTRODUCTION}

The accurate determination of spatially continuous pressure and temperature distributions on aerodynamic surfaces is critical for the understanding of complex flow mechanisms and for comparison with computational fluid dynamics (CFD) predictions. Conventional pressure measurements are based on pressure taps and electronically scanned pressure transducers. While these approaches provide accurate pressure information, pressure taps are limited to providing data at discrete points. Moreover, the integration of a sufficient number of pressure taps on a surface can be time and labor intensive and expensive.

The ability to make an accurate determination of pressure and temperature distributions over an aerodynamic surface based on an emitted optical signal from a luminescent coating has recently been developed. ${ }^{1-12}$ Pressure sensitive paint (PSP) measurements exploit the oxygen $\left(\mathrm{O}_{2}\right)$ sensitivity of luminescent probe molecules suspended in gaspermeable binder materials. If the test surface under study is immersed in an atmosphere containing $\mathrm{O}_{2}$ (e.g. air), the recovered luminescence intensity can be described by the Stern-Volmer relationship ${ }^{13}$

$$
\frac{\mathrm{I}_{0}}{\mathrm{I}}=1+\mathrm{K}_{\mathrm{sv}} \mathrm{P}_{\mathrm{O}_{2}}
$$

where $\mathrm{I}_{0}$ is the luminescence intensity in the absence of $\mathrm{O}_{2}$ (i.e. vacuum), $\mathrm{I}$ is the luminescence intensity at some partial pressure of oxygen $\mathrm{P}_{\mathrm{O} 2}$, and $\mathrm{K}_{\mathrm{SV}}$ is the Stern-Volmer constant. Since it is a practical impossibility to measure $\mathrm{I}_{0}$ in a wind tunnel application, a modified form of the Stern-Volmer equation is typically used. This form replaces the vacuum calibration $\left(\mathrm{I}_{0}\right)$ with a reference standard

$$
\frac{\mathrm{I}_{\mathrm{REF}}}{\mathrm{I}}=\mathrm{A}(\mathrm{T})+\mathrm{B}(\mathrm{T}) \frac{\mathrm{P}}{\mathrm{P}_{\mathrm{REF}}}
$$

where $\mathrm{I}_{\text {REF }}$ is the recovered luminescence intensity at a reference pressure, $\mathrm{P}_{\mathrm{REF}}$. There are two methods for acquiring PSP data. The most common method used for data acquisition is a "steady-state" mode. During steady-state PSP experiments, $I_{\text {REF }}$ is typically acquired while the wind tunnel is off or at very low speed and $P_{\text {REF }}$ is the static pressure when no wind is applied. Thus $\mathrm{I}_{\mathrm{REF}}$ is referred to as the "wind-off" intensity. $\mathrm{I}$ is the recovered luminescence intensity at some pressure P. Since this data is collected at a specific condition in the wind tunnel, I is also referred to as the "wind-on" intensity. A and B are temperature dependent constants for a given PSP formulation and are usually determined before hand using laboratory calibration procedures.

The second method of PSP data acquisition is known as "lifetime-based" PSP. ${ }^{14-16}$ Lifetime-based PSP data 
acquisition has many potential advantages over standard intensity-based PSP techniques. While both require a ratio of two images to account for nonuniformities in illumination and/or paint application, lifetime-based PSP techniques acquire the reference image at condition. Not only does this minimize tunnel occupancy time (thus reducing overall testing costs for some wind tunnels), but also reduces errors in recovered pressure due to mis-registration between the aero-loaded and unloaded images and temperature differences between wind-off and wind-on conditions.

In the time-resolved lifetime-based technique, excitation of the PSP is accomplished using a modulated light source (e.g. laser, flash lamp, or pulsed LED arrays). Following excitation of the PSP, the excited state luminescence decay is collected using a fast-framing camera (intensified CCD camera). Typically, the decay is approximated by acquiring two images at different delay times following the pulsed excitation and integrating photons for fixed periods of time (i.e. gate widths) that have been predetermined to maximize the pressure sensitivity, as demonstrated in Figure 1. The first image (Gate 1) usually consists of a short gate width and is collected either during the excitation pulse or shortly after it ends. This can be thought of as the reference image because the excitedstate decay has the least pressure sensitivity. The second image (Gate 2) is taken at a later time after the excitation pulse and usually has a longer gate width, ensuring maximum pressure (and temperature) sensitivity. However, to ensure adequate signal-tonoise $(\mathrm{S} / \mathrm{N})$, each gate is typically acquired following multiple excitation pulses.

Recent advances in ultra-stable light emitting diode (LED) technology and interline transfer CCD (IT-CCD) architectures have been used to construct a lifetime PSP system. Using pulsed LED arrays to excite the paint and acquiring data with a non-intensified IT-CCD camera minimizes the two main sources of noise in conventional lifetime PSP systems. The LED arrays show a greater degree of stability than either flash lamps or lasers, and the IT-CCD does not suffer from the noise that can be attributed to the intensifier of an ICCD.

This paper presents a detailed description of the lifetime-based PSP system, including results obtained for several different PSP formulations. Data recovered from a recent wind tunnel test will also be presented comparing results obtained using the lifetime-based system with a standard intensity-based PSP technique. Finally, efforts are underway to optimize the system by investigating new methods for acquiring lifetime PSP data. One promising approach involves acquiring a third gate to enable the determination of both temperature and pressure distributions simultaneously. ${ }^{17}$ Preliminary results using this approach are discussed.

\section{EXPERIMENTAL}

Lifetime PSP system: The lifetime PSP system developed is based on a pulsed excitation source and a specially developed interline transfer CCD camera. The modulated excitation source selected for this system consists of super-bright light emitting diodes with specially designed circuitry that allows for overdriving and operation at high repetition rates. With the modulating circuitry, the LEDs are overdriven using approximately four times the maximum allowable steady-state current. The lamps can be operated with up to a $5 \%$ duty cycle, and with minimum pulse widths of about $1 \mu \mathrm{sec}$, allowing for full turn on of the LEDs. For most of the experiments described, the lamps were operated with a square-pulse modulation of $10 \mu \mathrm{sec}$ pulses at a rate of $5 \mathrm{kHz}$. The lamps are commercially available and come in a variety of sizes (number of LEDs in the lamp) and wavelengths (from $395 \mathrm{~nm}$ to $520 \mathrm{~nm}){ }^{18}$

The CCD camera chosen for the system was a specially designed interline transfer camera (Roper Scientific CoolSnap HQ) employing a hardware accumulator. The camera operates by masking every other line of the chip. This allows charge to be transferred quickly (200 ns transfer time) from the unmasked region to the masked region for either storage or readout. The active area of the CCD chip is 1396 x 1040 pixels and the digitizer operates at 12-bit resolution (up to 4096 gray levels). The camera is equipped with a fast electronic shutter capable of acquiring images at a rate as fast as 1 usec. The hardware accumulator allows for multiple light pulses to be added together achieving the required signal-to-noise (SNR) ratio needed for low-light techniques without the added noise attributed to standard intensifiers. The maximum number of light pulses that can be added together is 32,768 , limited by the 16-bit digitization of the accumulator.

PSP Formulations: Several different PSP formulations were tested to validate the lifetime-based PSP calibration. All tests were performed in a calibration chamber designed to operate from vacuum to $15 \mathrm{psi}$ absolute and from $-30^{\circ} \mathrm{C}$ to $100^{\circ} \mathrm{C}$.

FEM: The first PSP tested was based on a formulation developed at the NASA Langley Research Center and 
described previously. ${ }^{19}$ The oxygen-permeable layer is made from a co-polymer of trifluoroethylmethacrylate and isobutylmethacrylate, in which platinum mesotetra(pentafluorophenyl) porphine $[\mathrm{Pt}(\mathrm{TfPP})]$ is dissolved. The formulation can be used with a white basecoat in a standard two-coat application and has been modified to include $\mathrm{TiO}_{2}$ for single coat applications.

$\underline{F I B}$ : The second formulation tested was developed by the University of Washington ${ }^{20}$ and is commercially available from ISSI. ${ }^{18}$ Like FEM, this PSP uses $\mathrm{Pt}(\mathrm{TfPP})$ as the luminophore. However, the $\mathrm{Pt}(\mathrm{TfPP})$ is immobilized in a fluoroisobutylmethacrylate copolymer. It is typically applied in a two-coat application with a specially developed basecoat, but has recently been formulated for use as a single coat. ${ }^{18}$

Sol-Gel: The last formulation tested was formulated by immobilizing ruthenium bathophenanthroline (RuBath) in an inorganic silicon dioxide matrix formed using a sol-gel process. A prehydrolized silica solution (Silbond H5) is the precursor in which the RuBath and a small amount of base are added. During curing of the paint, the prehydrolized silica forms a threedimensional silicon dioxide network forming the oxygen-permeable layer. This formulation is typically applied as a two-layer application. A layer of FEM with $\mathrm{TiO}_{2}$ is applied to the model to act as a basecoat, followed by a thin layer of the sol-gel PSP.

Validation Test: A validation test of the new lifetimebased PSP system was conducted at the Subsonic Aerodynamic Research Laboratory (SARL) at WrightPatterson Air Force Base in Dayton, OH. The SARL facility has a test section of 7 feet $\mathrm{x} 10$ feet $(2.13 \mathrm{~m} \mathrm{x}$ $3.05 \mathrm{~m}$ ) and is capable of operating at speeds up to Mach 0.4. Additionally, there is 360 degrees of optical access and the walls are $65 \%$ windows. The model tested was a lambda wing configuration that was constructed from resin materials via stereolithography techniques. The model was painted with a single-coat FIB formulation and 4 four-inch $(10 \mathrm{~cm}) 405 \mathrm{~nm}$ LED pods were used for illumination. Intensity-based PSP was accomplished using a standard scientific-grade back-lit CCD camera with an active area of 512 x 512 pixels and a digital resolution of 16 bits. Exposure times for the intensity-based PSP measurements were 1.5 seconds, and 16 images were averaged per test point. Wind-off images were generally collected after running the test point in order to minimize temperature differences between wind-off and wind-on images. Lifetime-based data were collected using the same PSP application. The 405-nm LED pods were modified so that they could be overdriven with $10 \mu$ sec pulses at 5 $\mathrm{kHz}$. In order to acquire enough light in the 32768 pulses allowed by the hardware integrator, the $1396 \mathrm{x}$ 1040 pixel CCD was binned down to $368 \times 260$ (binning factor of 4 ).

\section{$\underline{\text { RESULTS AND DISCUSSION }}$}

System Validation Using Calibration Rig: Several different paint formulations were tested in a calibration rig to validate the lifetime-based PSP system. In order to ensure that the system is able to approximate a typical excited-state intensity decay curve, single-coat FIB paints were applied to aluminum coupons and calibrated from 10 psia to $14.7 \mathrm{psia}$ at $25^{\circ} \mathrm{C}$. $5 \mu \mathrm{sec}$ images were collected at varying delays from the end of the excitation pulse from a 405-nm LED pod. Normalized data for 10 psia and 14.7 psia are shown in Figure 2a, showing that the decay curves can be obtained, and as expected, the lifetime decreases with increasing pressure. Excited state decay curves were also collected for a RuBath sol-gel PSP formulation by collecting $2 \mu \mathrm{sec}$ images at various decay times from the end of the excitation pulse from a 470-nm LED pod. These data are shown in Figure $2 b$ and illustrate the system sensitivity to the significantly shorter lifetime behavior exhibited by the RuBath luminophore.

Calibrations of each PSP formulation were performed between 10 psia and 14.7 psia at various temperatures. These data are shown in Figure 3. Data analysis was accomplished by taking the same small region of interest (approximately 2000 square pixels) in each image and recording the average intensity for each gate at each condition. The data in the plot are simply the first gate (G1) divided by the second gate (G2). As no wind-off image should be required for data analysis, no data normalization was performed. These data show that the actual recovered G1/G2 values are a strong function of temporal gate width as well as the delay time for each gate. For each of the samples investigated, G1 images were acquired during the light pulse in order to maximize signal, with G2 image acquisition temporally delayed from the excitation pulse to maximize pressure sensitivity.

It should also be noted that the pressure calibrations still show temperature sensitivity. While lifetime-based PSP data acquisition will not be affected by temperature differences between the reference image and the pressure image (as they are both taken at condition), the temperature of the surface must still be known in order to use the correct calibration. Thus, 
either knowing the temperature or using an in situ calibration against surface pressure taps will still be required with a standard two-gate lifetime approach. However, acquiring both "reference" (G1) and "run" (G2) images at condition eliminates the primary temperature sensitivity error source due to the large difference in tunnel static temperature between windoff and wind-on operation. Moreover, the acquisition of both images under aerodynamic load minimizes or eliminates the need to invoke image photogrammetry in the data analysis to align the spatially distorted (i.e., aero loaded) wind-on image with the wind-off image of the unloaded model.

Validation Test at the SARL Wind Tunnel: Global intensity and lifetime-based PSP measurements were performed in the SARL facility on a lambda wing configuration model as shown in Figure 4. Camera and excitation sources were mounted above the tunnel as shown in Figure 4 (right). Multiple configurations of the model were tested. These configurations were achieved by varying inboard and outboard flaps $\left(+20^{\circ}\right.$, $0^{\circ},-20^{\circ}$, and combinations of these values). All configurations were run at two angles of attack $\left(12^{\circ}\right.$ and $20^{\circ}$ ) and at two speeds (Mach 0.2 and 0.4). Typical test condition sequences for a model configuration consisted of pitching the model to $12^{\circ}$ at Mach 0.2 , acquiring PSP data, then pitching the model to $20^{\circ}$ and acquiring PSP data. For Mach 0.4, the reverse was done; PSP data were acquired at $20^{\circ}$ then the model was pitched to $12^{\circ}$ for PSP acquisition. Wind-off images for the intensity method were generally collected in an idle state after the PSP data had been collected. Finally, after collecting wind-off data, model changes were conducted and the process repeated for the new configuration.

Several (19) registration marks were added to the model to facilitate registration of the wind-on image to the wind-off image (for the intensity method) and to convert the two-dimensional image coordinates to three dimensions for in situ calibration against the existing pressure taps (the three-dimensional coordinates of the registration marks as well as the pressure taps were measured using a FaroArm coordinate mapper system). ${ }^{21}$ After registering the images (for the intensity method) and ratioing the pressure image with the reference image, the PSP was calibrated using six disparate pressure taps. All image analysis was accomplished using Greenboot ${ }^{21}$ running on a Linux platform.

A comparison of data collected using each technique is shown in Figure 5. The intensity-based method was used first and the lifetime data was acquired four days later from the same PSP application. To account for changes in the static and atmospheric pressures, the data has been converted to $C_{P}$. These data show that the lifetime-based method recovers similar qualitative and quantitative data as the intensity-based method. Moreover, the lifetime data was acquired without a wind-off image, and with no registration between the pressure image and reference image. Data analysis was accomplished by simply dividing the first gate image by the second gate image. There are slight differences between the two methods. Both methods are able to detect the vortex that forms at the nose of the model and its interaction with the wing-body junction vortex. However, there are slight differences in the recovered pressures at the leading edge of the wing. It is in this region that an oil deposit formed on the PSP, lowering its sensitivity to oxygen in this region. This oil can be seen in the photograph of the model taken just after the lifetime images were acquired and shown in Figure 6. It also bears noting that a $4 \times 4$ pixel binning (i.e., 16 pixels collapsed to one) process was required for acquisition of the lifetime images to compensate for the limited optical excitation power provided by the four, 4-inch LED arrays employed. This limitation in the spatial resolution recovered in the lifetime images can be easily overcome by integrating a greater number of pulsed LED illumination sources. Overall, this validation test has shown that with adequate pressure changes on a model, lifetime-based data acquisition techniques can recover similar information as conventional intensitybased methods without the need of shutting off the wind tunnel to acquire reference images.

Advanced Lifetime Imaging: While employing lifetime-based imaging can reduce the amount of wind tunnel residency time required to complete tests, it still suffers from errors due to the temperature sensitivity of the paint (see Figure 2). One promising method for alleviating this temperature sensitivity is to acquire a third gate. In this approach, a reference gate is acquired in addition to two different gates during the PSP intensity decay, as illustrated in Figure $7 .{ }^{17}$ If the gates are chosen such that the $\mathrm{G} 1 / \mathrm{G} 2$ ratio has a different pressure and temperature sensitivity relative to the G1/G3 ratio, then it is theoretically possible to measure the temperature and pressure simultaneously.

This three-gate approach to lifetime PSP was tested using both FEM and FIB painted coupons. Pt(TfPP)based PSPs were chosen because the longer excitedstate lifetime of the luminophore allows for a greater width and separation of the longer gates; thereby, 
maximizing the difference in the pressure and temperature sensitivities. For all experiments, gate delays were measured from the end of the excitation pulse. Gate 1 was chosen to be $0-1 \mu$ sec, Gate 2: 12-20 $\mu \mathrm{sec}$, and Gate 3: 30-83 sec. $^{17}$ The differences in the pressure and temperature sensitivities of G1/G2 and G1/G3 for each paint are shown in Figure 8.

The fitting model to calculate pressure based on temperature and $\mathrm{G} 1 / \mathrm{G} 2$ or $\mathrm{G} 1 / \mathrm{G} 3$ was assumed to be ${ }^{17}$

$$
\begin{aligned}
& P=\sum_{i, j=1}^{3} a_{i j}(G 1 / G 2)^{i-1} T^{j-1} \\
& P=\sum_{i, j=1}^{3} b_{i j}(G 1 / G 3)^{i-1} T^{j-1}
\end{aligned}
$$

where $\mathrm{P}$ and $\mathrm{T}$ are the pressure and temperature, respectively, and $\mathrm{a}$ and $\mathrm{b}$ are $\mathrm{a}$ set of calibration coefficients that must be determined for each paint in laboratory experiments. These coefficients were determined using a least-squares regression of calibration data acquired at different pressures and temperatures.

After collecting the three gates, temperature and pressure can be calculated by solving the following quadratic equation for $\mathrm{T}$

$$
\sum_{i, j=1}^{3} a_{i j}(G 1 / G 2)^{i-1} T^{j-1}=\sum_{i, j=1}^{3} b_{i j}(G 1 / G 2)^{i-1} T^{j-1}
$$

and then substituting into one of the original equations and solving for $\mathrm{P}$. The efficacy of this technique was demonstrated in the calibration rig and the results are shown in Figure 9. The apparent spread in the data is most likely due the lower dynamic resolution of the camera (12 bits). Further validation tests are underway to optimize this technique, but the results demonstrate the feasibility of this approach for the recovery of quantitative global pressure and temperature distributions in a single measurement under controlled environments.

\section{$\underline{\text { SUMMARY }}$}

A lifetime-based PSP system based on pulsed LED lamps and a new interline transfer CCD camera has been described. This system is able to recover both long (Pt(TfPP) and short (RuBath) excited-state lifetimes, spanning the gamut of currently available PSP formulations.

This system was demonstrated in a low-speed wind tunnel test at the SARL facility at WPAFB. Data recovered using the lifetime-based PSP system was both qualitatively and quantitatively similar to that acquired using a conventional intensity-based PSP approach. The differences between the two techniques can be attributed to debris sedimentation that occurred after the intensity-based method was used and before the lifetime-based method was deployed. This test further demonstrated that it possible to acquire data with this system without using conventional wind-off measurements and without errors that can be introduced with image registration and photogrammetry techniques. And in doing so, extend PSP applicability to models of high geometry (e.g., fighter configurations with stores) that are typically suffer errors due to shadows of external components on the main body.

Finally, an advanced technique is being explored that will allow for the simultaneous determination of both pressure and temperature. This requires the use of a third gate chosen so that pressure and temperature sensitivities of the $\mathrm{G} 1 / \mathrm{G} 2$ and $\mathrm{G} 1 / \mathrm{G} 3$ ratios are substantially different. This has been demonstrated in a calibration rig with facility validation targeted for the near term.

\section{ACKNOWLEDGEMENTS}

The authors would like to than Tim Bencic of the NASA Glenn Research Center and Dr. James Bell of the NASA Ames Research Center for very helpful discussions on employing the CoolSnap HQ camera. We would also like to thank John Davis, Jim Crafton, and Larry Goss from ISSI for discussions involving pulsed LED lamps and for assistance and providing single-coat FIB for the validation test in SARL. Finally, the validation test in SARL would not have been possible without the assistance of Charles Tyler, Gary Dale, and Thomas Tighe from AFRL/WPAFB, whose leadership and advocacy made it possible to collaborate under sanction of NASA Langley Research Center Interagency Agreement IA1-568, Annex 4.

\section{$\underline{\text { REFERENCES }}$}

1. Kavandi, J., et al., 1990, "Luminescence Barometry in Wind Tunnels," Rev. Sci. Instrum., Vol. 61, No. 11, 3340.

2. Morris, M.J., Benne, M.E., Crites, R.C., and Donovan, J.F., 1993, “Aerodynamic Measurements 
Based in Photoluminescence," AIAA Paper 930175 .

3. McLachlan, B. and Bell, J., 1995, "PressureSensitive Paint in Aerodynamic Testing," Experimental Thermal and Fluid Science, Vol. 10, 470.

4. Liu, T., Campbell, B., Burns, S., and Sullivan, J., 1997, "Temperature- and Pressure-Sensitive Luminescent Paints in Aerodynamics," Applied Mechanics Review, Vol. 50, No. 4, 227.

5. Asai, K., Kanda, H., and Kunimasu, T., 1996, "Detection of Boundary-Layer Transition in a Cryogenic Wind Tunnel by Using Luminescent Paint," AIAA Paper 96-2185.

6. Asai, K., et al. "Surface Pressure Measurement in a Cryogenic Wind Tunnel by Using Luminescent Coating," ICIASF, Monterey, CA, 1997.

7. Shimbo, Y., Mehta, R., and Cantwell, B., 1997, "Vortical Flow Field Investigation Using the Pressure Sensitive Paint Technique at Low Speed," AIAA Paper 97-0388.

8. Torgerson, S., Liu, T., and Sullivan, J., 1996, "Use of Pressure Sensitive Paints in Low Speed Flows," AIAA Paper 96-2184.

9. Carroll, B., Abbitt, J. Lukas, E., and Morris, M., 1995, "Pressure Sensitive Paint Response to a Step Pressure Change," AIAA Paper 95-0483.

10. Winslow, N., Carroll, B., and Setzer, F., 1996, "Frequency Response of Pressure Sensitive Paints," AIAA Paper 96-1967.

11. Coleman, P.B., Wallis, S.B., Dale, G.A., Jordan, J.D., Watkins, A.N., Goss, L., Davis, J.C.P.N., and Walter, T.M., 2002, "A Comparison of Pressure Sensitive Paint (PSP) Techniques for Aerodynamic Testing at Slow Velocities," Society for Automotive Engineers Paper 02B-29.

12. Jordan, J.D., Watkins, A.N., Davis, J., Weaver, W.L., Dale, G.A., Navarra, K., Urban, J., Devoid, W., and Stange, R., 1999, "Pressure-Sensitive Paint Measurements in a Large Scale Commercial Engine Test Stand," Proceedings of the $18^{\text {th }}$
International Congress on Instrumentation in Aerospace Simulation Facilities.

13. Lakowicz, J., Principles of Fluorescence Spectroscopy (Plenum Press, New York, 1983).

14. Engler, R., and Klein, C., 1997, "DLR PSP System: Intensity and Lifetime Measurements," Proceedings of the $17^{\text {th }}$ International Congress on Instrumentation in Aerospace Simulation Facilities.

15. Holmes, J., 1998, "Analysis of Radiometric, Lifetime and Fluorescent Imaging for Pressure Sensitive Paint," Aeronautical Journal, 0001-9240, Vol. 102, pp. 189-194.

16. Bell, H.J., Schairer, T.E., Hand, A.L. and Mehta, R.D., 2001, "Surface Pressure Measurements using Luminescent Coatings," Annu. Rev. Fluid Mech., Vol. 33, pp. 115-206.

17. Mitsuo, K., Egami, Y., Asai, K., Suzuki, H., and Mizushima, H., 2002, "Development of Lifetime Imaging System for Pressure-Sensitive Paint," AIAA-2002-2909.

18. Pressure sensitive paint and PSP hardware, software, and services are commercially available from Innovative Scientific Solutions, Inc. (ISSI), located in Dayton, OH. Their web address is www.innssi.com.

19. Oglesby, D.M. and Upchurch, B.T., 1999, "In Pursuit of the Ideal Pressure Sensitive Paint," Proceedings of the $7^{\text {th }}$ Annual Pressure Sensitive Paint Workshop, West Lafayette, IN.

20. Puklin, E., Carlson, B., Gouin, S., Costin, C., Green, E., Ponomarev, S., Tanji, H., and Gouterman, M., 2000, "Ideality of Pressure Sensitive Paint. I. Platinum Tetra(pentafluorophenyl)porphine in Fluoroacrylic Polymer," J. Appl. Polym. Sci., Vol. 77(13), pp. 2795-2804.

21. Bell, J., and McLachlan, B., 1996, "Image Registration for Pressure-Sensitive Paint Applications," Experiments in Fluids no. 22, pp. 78-86. 


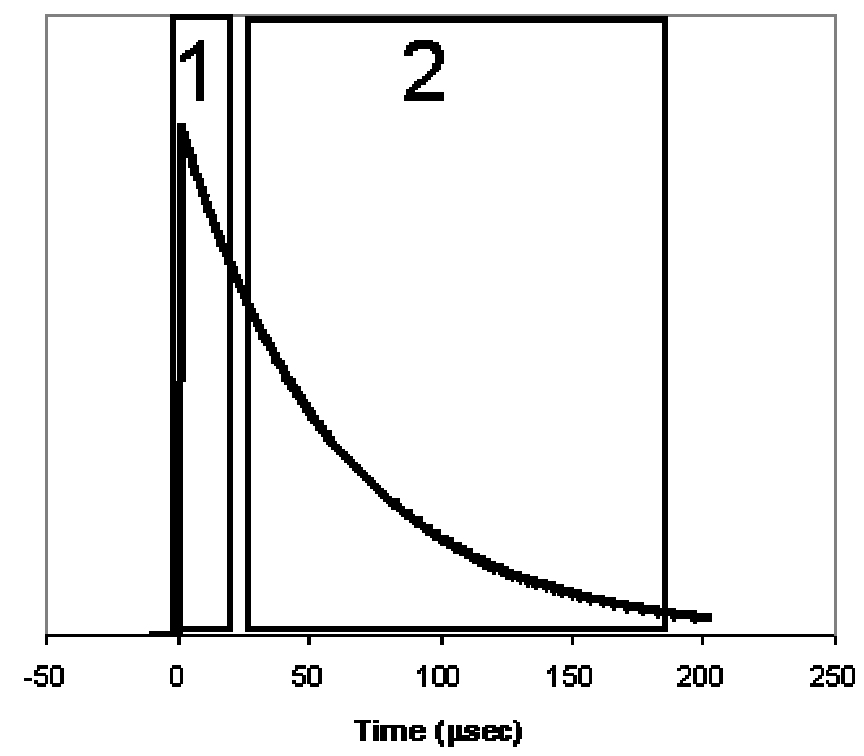

Figure 1. Standard two-gate lifetime technique. The numbers represent the respective gates acquired for data reduction.
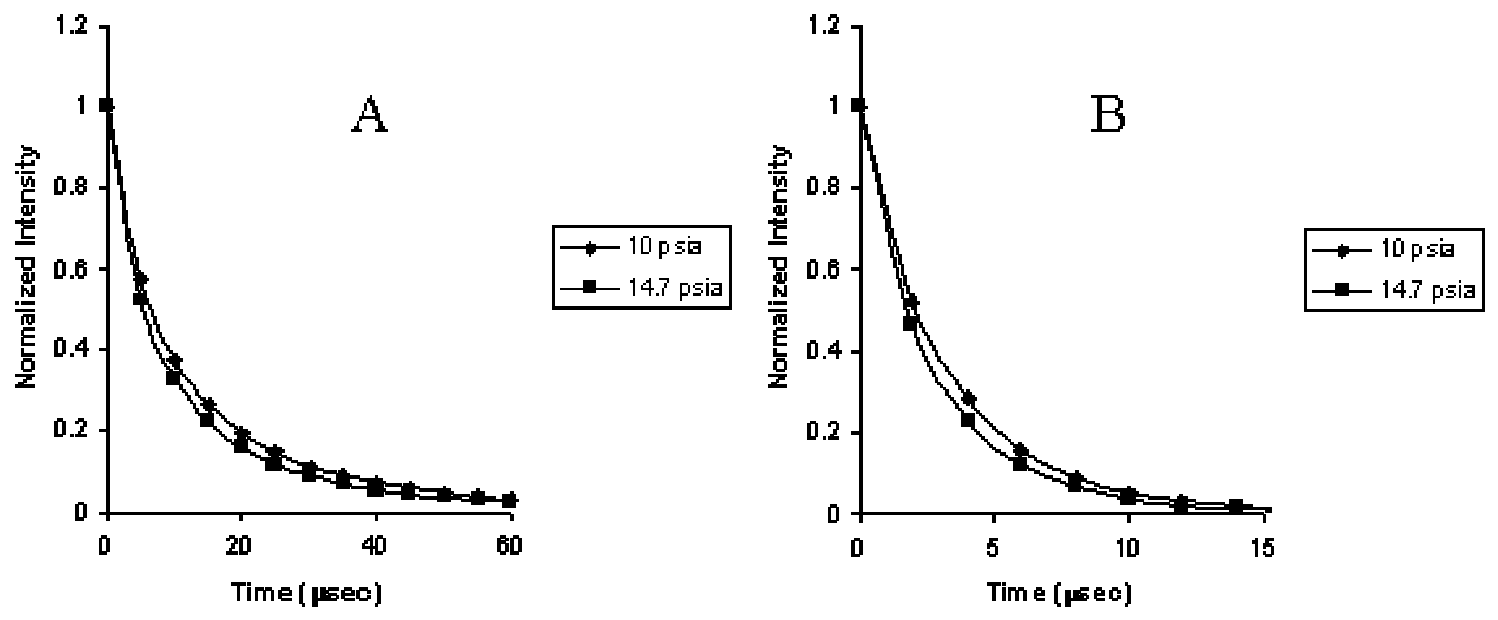

Figure 2. Excited-state decays recovered using the lifetime PSP system. (A) Single-coat FIB PSP decay collected by taking $5 \mu \mathrm{sec}$ pulses at various delays from the end of the excitation pulse. (B) RuBath solgel PSP decay collected by taking $2 \mu \mathrm{sec}$ pulses at various delays from the end of the excitation pulse. 

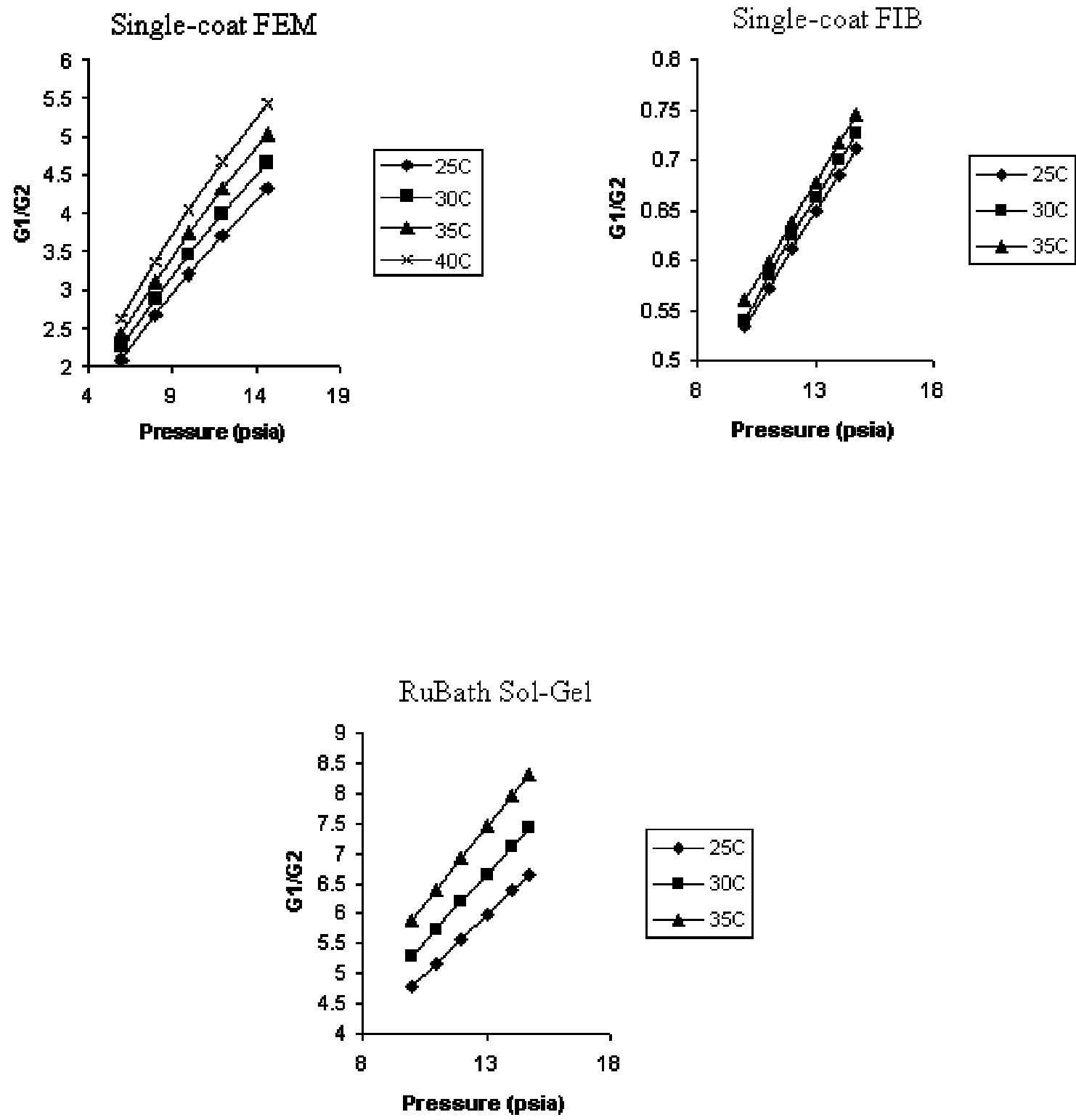

Figure 3. Lifetime calibration for various PSP formulations. No normalization of the data has been performed to fully simulate data collection in the wind tunnel (reference images taken at condition, not wind-off). 




Figure 4. (Left) Lambda wing configuration mounted in the SARL facility. (Right) PSP lifetime system mounted above the tunnel.

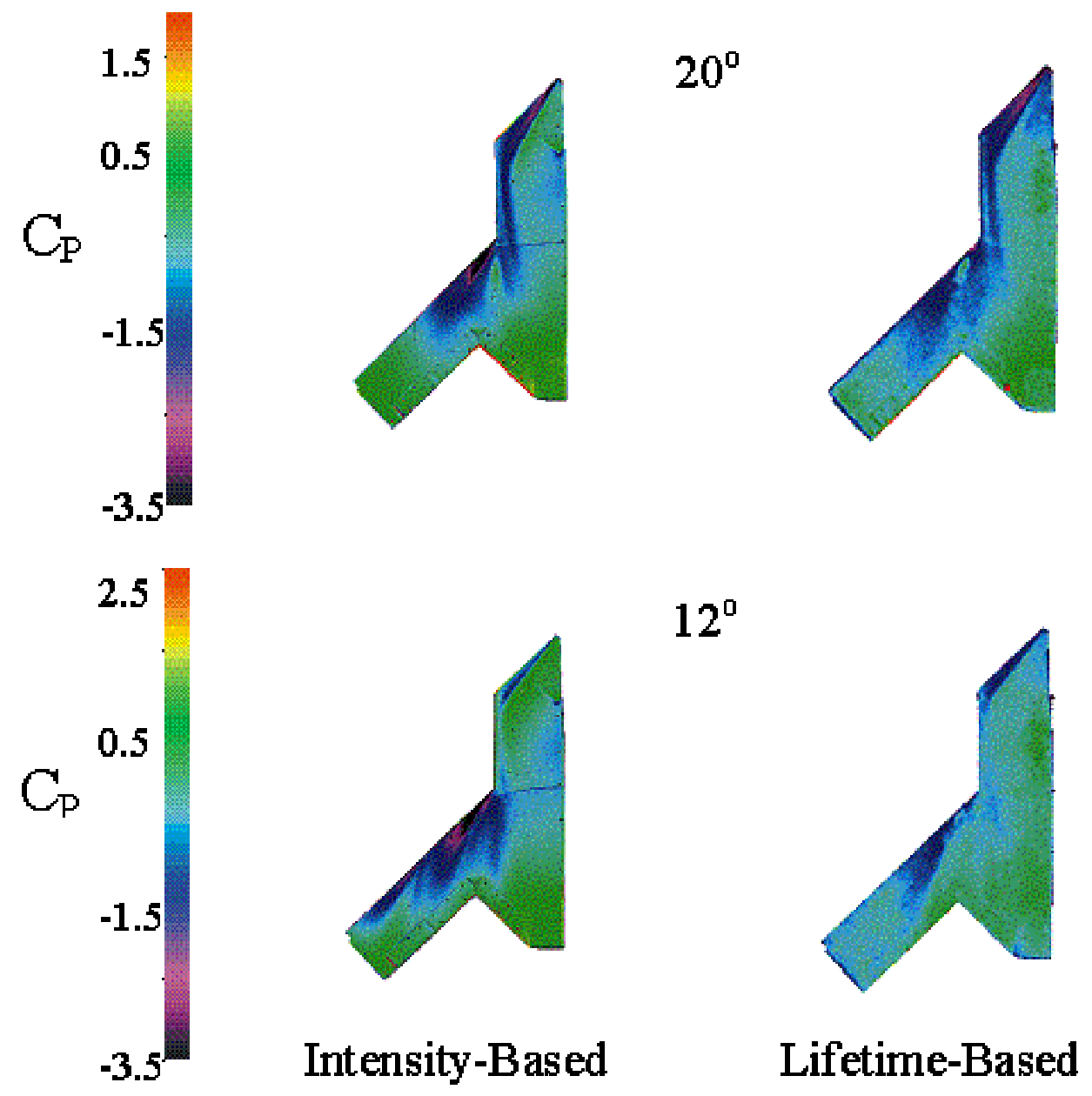

Figure 5. Comparison of PSP data from the lambda wing model collected using the intensity-based method and the lifetime-based method. Data has been normalized to $C_{P}$ to account for differences in static and atmospheric pressure. 


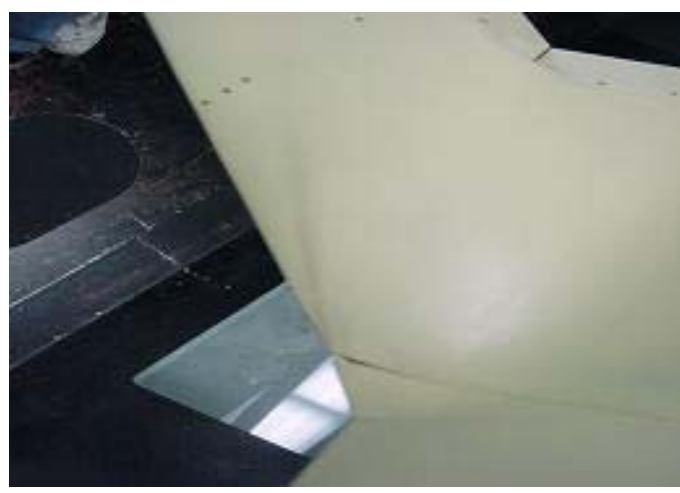

Figure 6. Photograph of lambda wing model showing oil sedimentation on the leading edge.

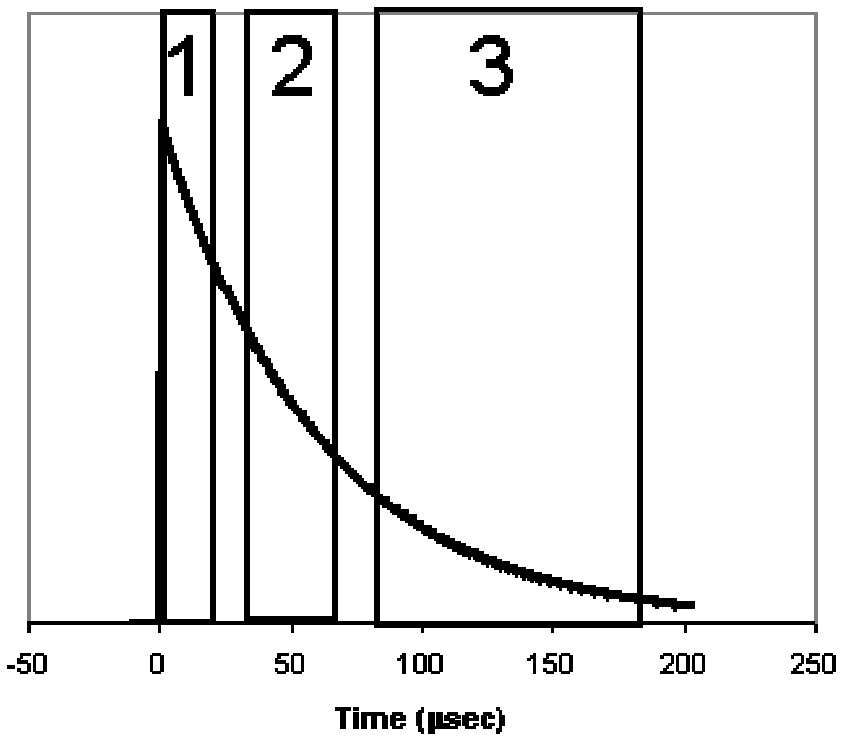

Figure 7. Three-gate lifetime technique for determining pressure and temperature simultaneously. The numbers represent the respective gates acquired for data reduction.
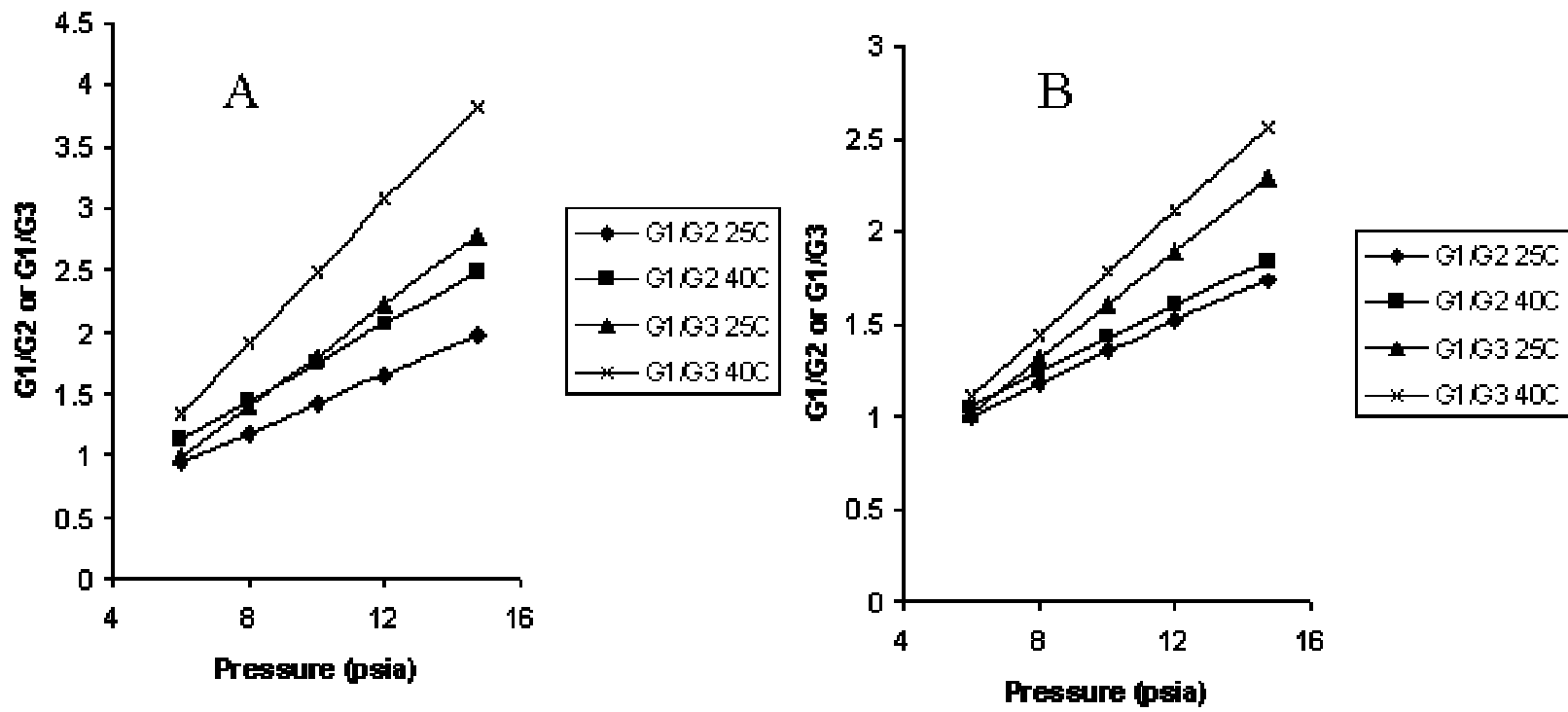

Figure 8. Calibrations showing different temperature and pressure sensitivities for G1/G2 and G1/G3. (A) Single-coat FEM. (B) Single-coat FIB. 

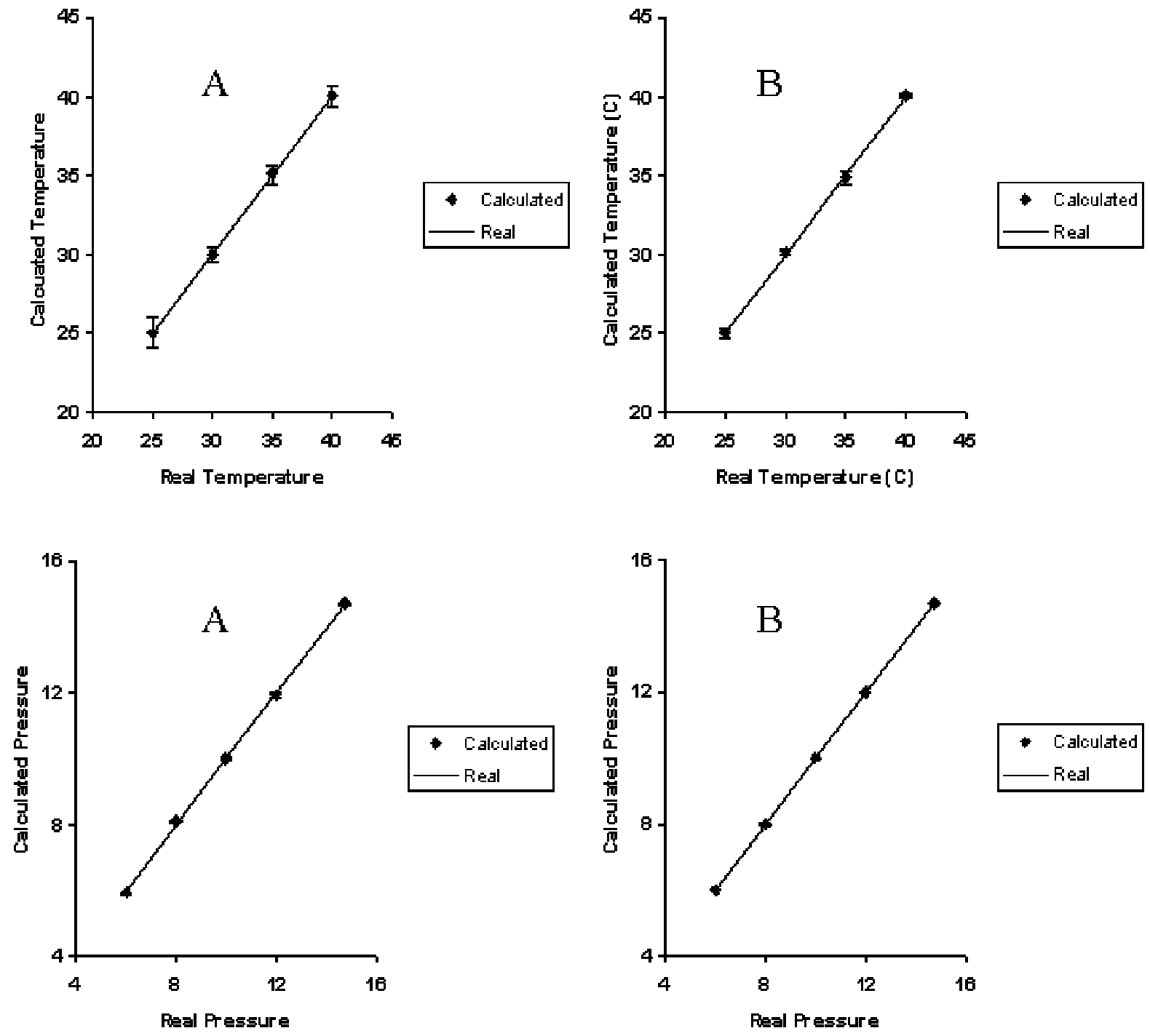

Figure 9. Calculated vs. recovered temperature (Top) and pressure (Bottom) for single-coat FEM (A) and single-coat FIB (B) PSP formulations. 


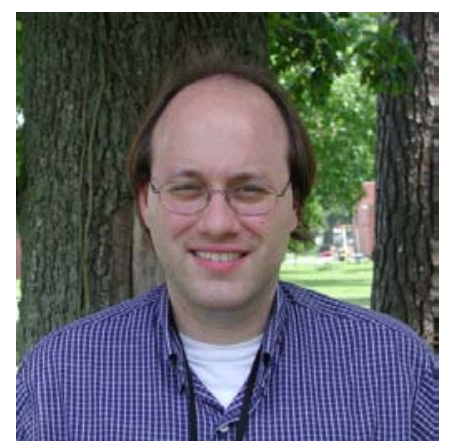

Neal Watkins received his B.S. degree in Chemistry from Christian Brothers University in 1992 and his Ph.D. in Analytical Chemistry from the University of Arkansas in 1996. Dr. Watkins' doctoral research focused on creating chemical and biochemical sensors using quartz crystal microbalance technology. His interest in optical systems and sensors came about while performing postdoctoral work at the University of Buffalo and the Naval Research Laboratory in Washington, DC. He joined the Innovative Scientific Solutions, Inc. (ISSI) in 1998, where he performed research in the area of pressure sensitive paints and related systems, eventually leading the research and commercialization efforts. In 2000, Dr. Watkins joined the Advanced Chemistry Group at the NASA Langley Research Center leading its research and development in the areas of PSP, low-temperature oxidation catalysts for environmental remediation, and using nanotechnology to create novel sensors and materials. 